\title{
Design and Analysis of Variable Gain Amplifier with Erbium -Doped Fiber Switching
}

\author{
Shikha Gautam ${ }^{1}$, Prof. R.L. Sharma ${ }^{2}$ \\ Department of Electronics \& Communication Engineering \\ Ajay Kumar Garg Engineering College, Ghaziabad
}

\begin{abstract}
Design and analysis of Variable gain amplifier with Erbium doped fiber range with the switches by using the WDM technology and a small noise figure (NF) and increased Variable Gain. The EDFA can change the total erbium-doped fiber length by selecting many combinations of five EDFs with optical switches (SWs) and WDM technology. Fundament and principle of the EDFA is based on the spontaneous emission absorption energy levels method. Design and analysis of the numerical calculation of the NF and the pump power using the analytical model of an EDFA indicates that such a variable gain EDFA has an advantage over a conventional multi stage EDFA in terms of Gain variation and required pump power. These characteristics are demonstrated by constructing a variable gain EDFA that employs SWs and variable optical attenuators (VOAs) by using the MATLAB. The variable gain EDFA exhibits a smaller NF variation of $6.5 \mathrm{~dB}$ for the $85 \mathrm{~dB}$ variable gain range than conventional and more EDFAs amplifiers.
\end{abstract}

Key words- Erbium doped fiber amplifier (EDFA), Wavelength division multiplexing (WDM), and Optical fiber length, VOA (variable optical attenuator), SWs (optical switches).

\section{INTRODUCTION}

In optical communication network, signals travel through fiber for very long distances in the form of laser [1]. An optical amplifier is a device that amplifies an optical signal directly, without the need to first convert system in to an electrical signal automatically. Optical fiber amplifiers provide the amplification of optical signals by effecting stimulated emission of photons by rare earth element ions implanted in the core of the optical fiber cable. Erbium type of material is preferred rare earth element for this purpose [2]. EDFAs are used to provide amplification in long distance optical communication with fiber low loss at higher power and wavelengths. High power semiconductor laser diode is very useful to provide the light to pump EDFAs.

The EDFA was the first successful optical amplifier that was restructured the optical communication industry in the early 1990. EDFAs are used with the wavelength division multiplexed (WDM) systems [2] [3]. WDM technology employing erbium-doped fiber amplifiers (EDFAs) provides a stage for purposeful improvement in network bandwidthcapacity. WDM will provide a dominant role to the next generation of high-speed networks. When the number of WDM channels transmitted through a network varies due to network reconfiguration or channel failure, number of channel increment or decrement, it is depend on the channel will tend to perturb signals at the surviving channels that share all or part of the route.

An Amplifier configuration is by using the WDM technology in Cascade method. Implementing a WDM system including the EDFA does not have to amplify the wavelength of the channels equally and frequently to have equalized gain spectra in order to obtain uniform output powers and similar signal to noise ratio. There are several methods in designing a flat spectral gain EDFA such as by controlling the doped fiber length and the pump power, reducing the noise figure and increased the gain of the signal laser. WDM technology also using by the switching technology, switching technology used to increase the area and network of the system and more router connect with the optical fiber cables.

To propose EDFAs with the optical amplifier communication system, we have to utilize EDFAs with WDM technology with the using switching to provide improved gain. The W-EDFA employs by using the SWs and VOAs, Software using Matlab to change the ways in which EDFs combine. First we describe the principle of EDFA, fundamental and working and concept of EDFA. Secondly, describe the design of the SW-EDFA with WDM. We compare the SW-EDFA experimentally with a conventional variable gain EDFA in terms of NF and required pump power by using an analytical model of the EDFA. Since the SW-EDFA can have many different EDF combinations, the pump power requirement of the EDFA will differ from the variable gain in, which also clarifies the pump power needed to obtain variable gain, with the using convolution method by using more number of EDFAs with VOA, this paper III Section. IV, we describe the Numerical and theoretical configuration of EDFA gain characteristics with the wavelengths. In this section we increased the gain by using the different wavelengths and pump power and gain value is changed. In V, Section Experimental Result and Discussions are with the using of wavelength and different pump power. Experimental set up shown the NF is reduced and gain of the design is increased. The main conclusions are summarized result of this paper in Section VI. Analysis 
and design of configuration by the EDF is improved the gain of the fiber.

\section{FUNDAMENTAL OF EDFA}

An erbium-doped fiber is an optical fiber of which the core is doped with rare-earth element erbium ions Er3 that is rare earth material [3]. A configure energy level diagram of Er3+ ion is shown in Figure 1. When a $974 \mathrm{~nm}$ pump laser diode beam is fed into an erbium-doped fiber, Er3+ will be excited from the ground state E1 (Lower Level) to the higher level E3. The excited Er3+ ions on E3 Level will rapidly decay to energy level (Lower) E2 through non radioactive emission. The excited ions on E2 Level eventually return to ground state E1 Level through spontaneous emission, which produces photons in the wavelength band $1520-1570 \mathrm{~nm}$ (C band). The spontaneous emission will be amplified as it propagates through the fiber, especially when the pump laser power is increasing rapidly. As amplified spontaneous emission (ASE) covers a wide wavelength range 1520-1570nm, we can use it as a broadband light source also. EDFA principle based on the ASE fundamentals [7].

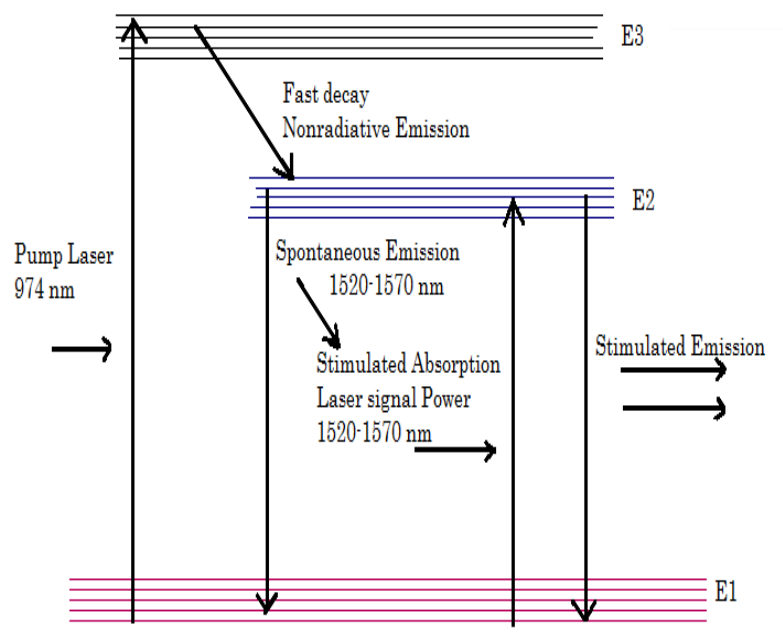

Figure 1: Structure of the System

If a laser signal with a wavelength between 1520 and $1570 \mathrm{~nm}$ or $\mathrm{C}$ band, and a 974 pump laser are fed into an erbium-doped fiber simultaneously as shown in Figure 1, there are three possible outcomes for the signal photon: (i) stimulated absorption, signal photon excites an erbium ion from the state E1 to a higher level E2 and become annihilated in the process; (ii) stimulated emission, signal photon stimulates an erbium ion at state E2 to decay to E1, Producinganother identical photon. Thus the signal is amplified by this. (iii) Signal photon can propagate through the fiber. In the spontaneous emission always occurs between level E2 and level E1. When pump laser power is high than the population inversion is achieved between the energy level E2 and E1 of erbium-doped fiber, the input laser signal passing through the fiber is then be amplified also. Thus we can use erbium-doped fiber and pump laser to construct an opticalamplifier, which called erbium-doped fiber amplifier.

The Fundamental of the EDFA shown in Figure 1, than basic configuration for the EDFA with an optical fiber is shown in Figure 2 An EDFA existed of a short length of optical fiber whose core has been doped with less than 0.1 $\%$ of erbium, an optically active rare earth element (erbium) that has many unique intrinsic properties for optical amplification. For example, the erbium atom has a Meta stable state with the remarkable long lifetime. This makes it possible to obtain an optical gain which takes a long time to saturate, and increased the gain also. The EDF is compatible with conventional fiber system and may be fusion spliced to other components. The signal Power and pump Power are combined and through a WDM coupler and launched into an erbium doped. Its core is the erbium doped optical fiber system with the Erbium type material, which is typically a single-mode fiber. Pump light propagating along the EDF is depleted as erbium ions are raised to an excited state (higher level of energy). As the signal propagates in the EDF, it stimulates emission of light from the excited ions, there by amplifying the signal power by using EDF. Construction of a typical EDFA module is shown in Figure 2. The erbium-doped fiber (EDF) is compatible with conventional fiber and may be fusion spliced to other components also. EDFA is constructed by fusion splicing discrete fiber pigtailed component. EDF amplifier configurations with Pump the isolator at the input prevents light originating from amplified spontaneous emission from disturbing any previous stages, whereas that at the output suppresses shortly, if output light is reflected back to the amplifier. Without isolators, fiber amplifiers can be sensitive to backreflections of the laser. There are optical isolators in input, various other components can be also contained in a commercial fiber amplifier For example, fiber couplers and photo detectors for monitoring optical power level, pump laser diode with control electronics and gain-flattening filter.

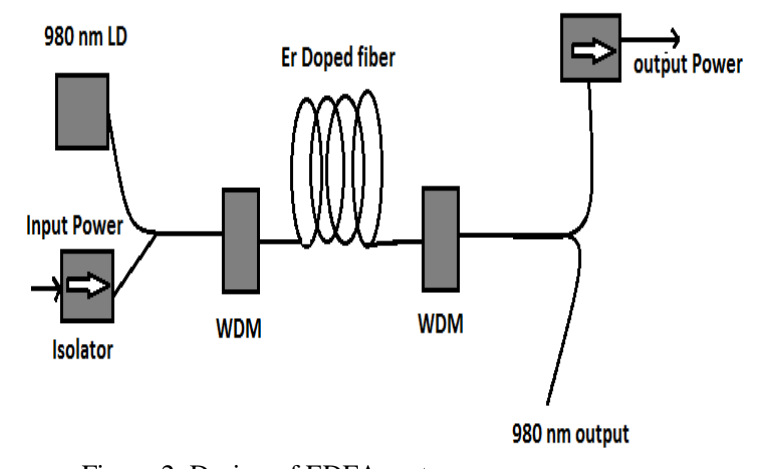

Figure 2: Design of EDFA system

In order to pump the erbium ions up to an upper energy level, there are several pump wavelength bands. At present, $1480 \mathrm{~nm}$ and $980 \mathrm{~nm}$ high power laser diodeshave proved to be the two most efficient pump wavelengths. 


\section{DESIGN AND ANALYSIS}

An SW-EDFA consists of multiple amplifier stages, SWs, and VOAs, and each amplifier stage has one EDF as an amplification medium. Changing the direction of the optical switches changes the active EDF combinations [13]. The different combinations with different discrete EDF lengths change the amplifier gain discretely. The VOAs adjust the gain so that a continuous gain is obtained between the discrete gains. Figure 3 shows the concept of the SW-EDFA (Switching Erbium doped fiber amplifier) with VOAs, An SW-EDFA consists of multiple amplifier stages, SWs, and VOAs, used for the amplification medium, and make a large network. The different combinations with different discrete EDF lengths change the amplifier gain and noise figure also and by using the switching the coverage area is increase. The VOAs adjust the gain so that a continuous gain is obtained between the discrete gains. The SW-EDFA varies the total EDF length by using switching the signal route and EDF combination also.

The combination of the 5 EDF amplifiers with switches is shown by the figure 3 . There are combination of the switches increase the length of the fiber and increase of the network area. The main concept is that number of amplifiers increased the losses of the fiber but by using the EDF amplifiers reduced the losses also and increased the gain of the system. $2 \times 2,2 \times 3,1 \times 3,4 \times 1$ type of switches combination used in this design. At the different wavelength and pump power to find the values of the gain and the noise figure. Large Network is cover the more area and more amplifiers produced the high gain and reduced the NF.

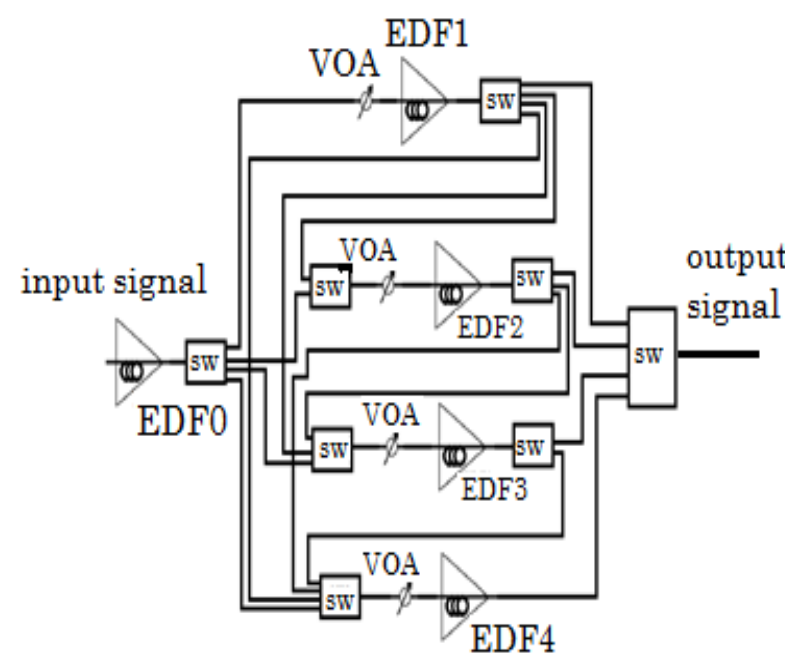

Figure3: Design of EDFA with Switches Model

\section{DESIGN AND ANALYSIS EDFA GAIN CHARACTERSTIC}

In this paper we calculate the NF and the pump power with the different wavelength for an SW-EDFA with the configuration shown in Fig. 3 and compare them with those for conventional system, by using two-stage , three-stage and four- EDFAs cascade systems. The conventional EDFAs consist of multiple amplifier stages and VOAs located with the every amplifier, and achieve a high variable gain by adjusting the attenuation of those VOAs. The calculation parameters for EDF are listed in Table I. The EDF used in the calculation is assumed to be an Al-Ge-Er-doped fiber [10], and design parameters for the simulation. EDF lengths of amplifier stages 0, 1, 2, 3 and 4 of the SW-EDFA are 8.2, 6.8, 9.5,4.2 $\mathrm{m}$ and $2.2 \mathrm{~m}$ respectively. The signal routes with switches for different EDF combinations are shown in Table II, the range of the combination of the routers also shown in table III. The EDF length of the first amplifier stage of the conventional two- and four-stage EDFAs are set at the same length as stage 0 of the SW-EDFA. The second amplifier stage of the two-stage EDFA has a $10.4 \mathrm{~m}$ long EDF. The remaining amplifier stages of the four-stage EDFA all have $19.2 \mathrm{~m}$ long EDFs. Each EDF is forward by the pumped power with $980-\mathrm{nm}$. The main aim of the paper to reduce the NF and increased the Gain of the input signal power.

Table I: Simulation Parameters for Design

\begin{tabular}{|c|c|c|}
\hline $\begin{array}{c}\text { N } \\
\text { o }\end{array}$ & Parameters & Range \\
$\mathbf{1}$ & Technology & Switching Technology \\
\hline $\mathbf{2}$ & $\begin{array}{c}\text { Wavelength } \\
\text { Range (nm) }\end{array}$ & $1536.61-1560.61$ \\
\hline $\mathbf{3}$ & $\begin{array}{c}\text { Pump } \\
\text { Wavelength }\end{array}$ & $980 \mathrm{~nm}, 1480 \mathrm{~nm}$ \\
\hline $\mathbf{4}$ & $\begin{array}{c}\text { Cut-off } \\
\text { Wavelength }\end{array}$ & $1400 \mathrm{~nm}$ \\
\hline $\mathbf{5}$ & $\begin{array}{c}\text { Pump Power } \\
\text { (MW) }\end{array}$ & $50-200$ \\
\hline $\mathbf{6}$ & $\begin{array}{c}\text { Noise Figure } \\
(\text { dB })\end{array}$ & $6-8$ \\
\hline
\end{tabular}

Table II: EDF combination of the amplifiers

\begin{tabular}{|c|c|}
\hline Combinations & Signal Routing \\
\hline A & EDF0 - EDF1 \\
\hline $\mathbf{B}$ & EDF0 - EDF2 \\
\hline $\mathbf{C}$ & EDF0 - EDF3 \\
\hline $\mathbf{D}$ & EDF0-EDF4 \\
\hline $\mathbf{E}$ & EDF0- EDF1 - EDF2 \\
\hline $\mathbf{F}$ & EDF0- EDF1 - EDF3 \\
\hline $\mathbf{G}$ & EDF0- EDF1 - EDF4 \\
\hline $\mathbf{H}$ & EDF0- EDF2 - EDF3 \\
\hline $\mathbf{I}$ & EDF0- EDF3 - EDF4 \\
\hline $\mathbf{J}$ & EDF0- EDF1 - EDF2- EDF3 \\
\hline $\mathbf{K}$ & EDF0- EDF1 - EDF2- EDF4 \\
\hline $\mathbf{M}$ & EDF0- EDF1 - EDF3- EDF4 \\
\hline $\mathbf{N}$ & EDF0- EDF2 - EDF3- EDF4 \\
\hline $\mathbf{P}$ & EDF0- EDF1 - EDF2- EDF3- EDF4 \\
\hline
\end{tabular}


Table III: Variable Gain range of the each EDF combination

\begin{tabular}{|c|c|c|}
\hline Combinations & Signal Routing & $\begin{array}{c}\text { Combination } \\
\text { Length }\end{array}$ \\
\hline $\mathbf{A}$ & EDF0 - EDF1 & $15 \mathrm{~m}$ \\
\hline $\mathbf{B}$ & EDF0 - EDF2 & $17.7 \mathrm{~m}$ \\
\hline $\mathbf{C}$ & EDF0 - EDF3 & $12.4 \mathrm{~m}$ \\
\hline $\mathbf{D}$ & EDF0-EDF4 & $10.4 \mathrm{~m}$ \\
\hline $\mathbf{E}$ & EDF0-EDF1 - EDF2 & $24.5 \mathrm{~m}$ \\
\hline $\mathbf{F}$ & EDF0-EDF1 - EDF3 & $19.2 \mathrm{~m}$ \\
\hline $\mathbf{G}$ & EDF0-EDF1 - EDF4 & $17.2 \mathrm{~m}$ \\
\hline $\mathbf{H}$ & EDF0-EDF2 - EDF3 & $21.9 \mathrm{~m}$ \\
\hline $\mathbf{I}$ & EDF0-EDF3 - EDF4 & $14.6 \mathrm{~m}$ \\
\hline $\mathbf{J}$ & EDF0- EDF1 - EDF2-EDF3 & $28.7 \mathrm{~m}$ \\
\hline $\mathbf{K}$ & EDF0- EDF1 - EDF2-EDF4 & $26.7 \mathrm{~m}$ \\
\hline $\mathbf{M}$ & EDF0- EDF1 - EDF3-EDF4 & $21.4 \mathrm{~m}$ \\
\hline $\mathbf{N}$ & EDF0- EDF2 - EDF3-EDF4 & $24.1 \mathrm{~m}$ \\
\hline $\mathbf{P}$ & EDF0- EDF1 - EDF2-EDF3-EDF4 & $30.9 \mathrm{M}$ \\
\hline
\end{tabular}

Figure 4 and 5 respectively, shows the amplifier dependence of the NF at the signal wavelength of 1530.33 $\mathrm{nm}$, at the two stages and four stages amplifier configuration. In Figure 4 shows that when noise figure is $13 \mathrm{~dB}$ then the gain is increase $35 \mathrm{mw}$. The total power of the signal is addition of the total amplifiers power so the power of the four stages is more than the two stages. In the graph 4.6 , noise figure is $9 \mathrm{~dB}$ then gain is $35 \mathrm{mw}$, it is shown that the NF is decreased than the Gain of the signal increased.

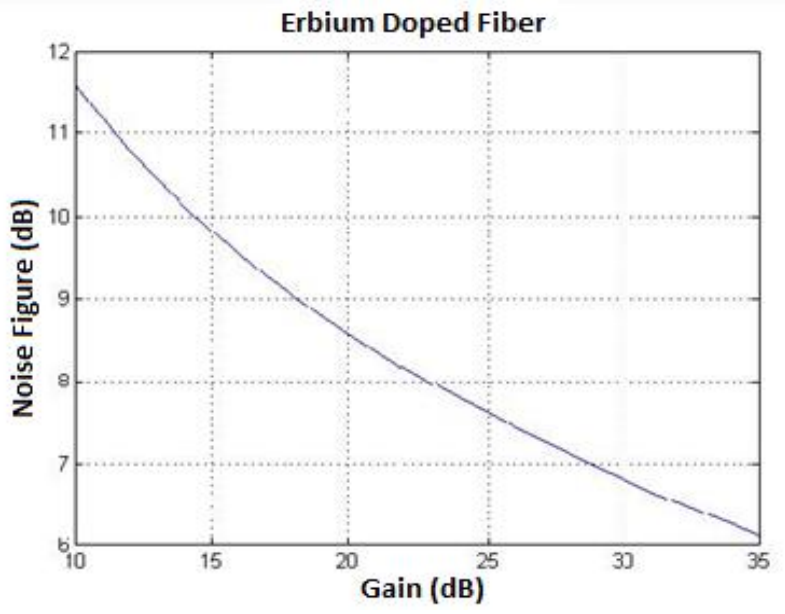

Figure 4: Graph between noise figure and Gain for stage 2

Figure 6 shows the configuration by using the switches. Combination of the five amplifiers with the using of switches power of the combination is increased. We are using the amplification gain is $10 \mathrm{~dB}$ and noise figure is $11.50 \mathrm{~dB}$ for Two stage amplifier. Noise Figure for Four stage amplifier is $8.5 \mathrm{~dB}$ and the Gain is the $10 \mathrm{~dB}$. In this configuration number of amplifiers are five switching EDFA noise figure is $7.8 \mathrm{~dB}$ with using gain is $10 \mathrm{~dB}$. Figure. 6 concludes that system performance is better for sw-edfa Respectively, Pump Power is $5.8 \mathrm{mw}$ and the Wavelength is $980 \mathrm{~nm}$.

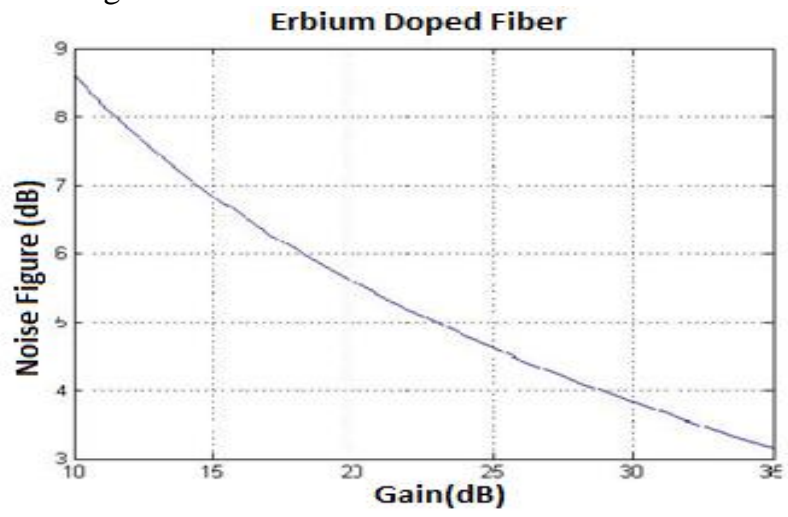

Figure 5: Graph noise figure and Gain for stage 4

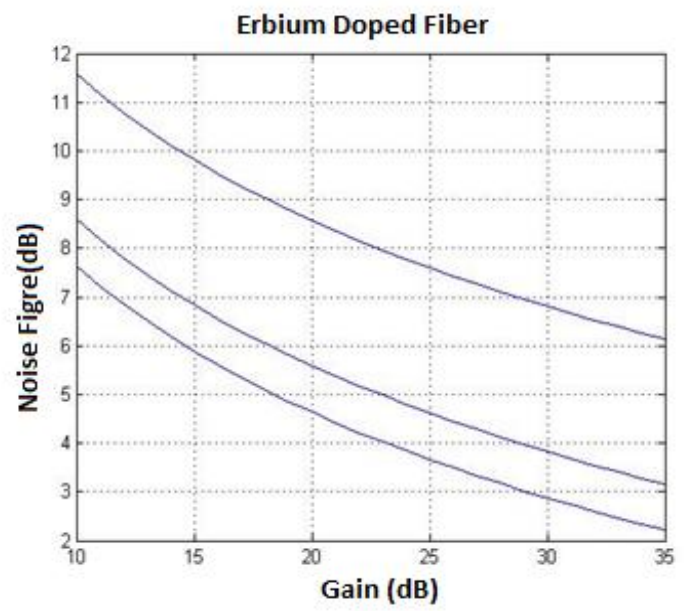

Figure 6: Graph between noise figure and pump power by using switches

\section{DISCUSSION OF POWER CONSUMPTION}

Figure.7 shows the amplifier gain dependence of the total pump powers and the conventional two- and four stage EDFAs without switches configuration. The amplifier Gain and wavelength by using pump power with different doping concentrations is shown in Figure 7. The Pump wave length is $976 \mathrm{~nm}$. Pump power for the amplifier is 50- $200 \mathrm{mw}$ and the doping of the system is $550 \mathrm{ppm}$. In this configuration shown that the pump power is increased than the Gain of the amplifier also increased at the different doping values.

Figure 8 shows that the Gain is against wavelength at fiber length $\mathrm{L}$ is $8.2 \mathrm{~m}$ and other fiber length $\mathrm{L}$ is $17 \mathrm{~m}$. When the length is $8.2 \mathrm{~m}$ gain is $20 \mathrm{~dB}$ and length is the $17 \mathrm{~m}$ then the gain is goes to $45 \mathrm{~dB}$, so by this shows when the length is increase then the gain is also increase of the fiber.

Figure 9 shows the amplifier dependence with the switches. Convolution method is using by the five EDF amplifiers with the switches and VOA, Figure 3 
shows the designing and combination of the EDF with the switches and VOA. The Gain of amplifier is the EDF0, EDF1, EDF2, EDF3 and EDF4 is $8.2 \mathrm{~dB}, 6.8 \mathrm{~dB}, 9.5 \mathrm{~dB}$, $4.2 \mathrm{~dB}$ and $2.2 \mathrm{~dB}$.

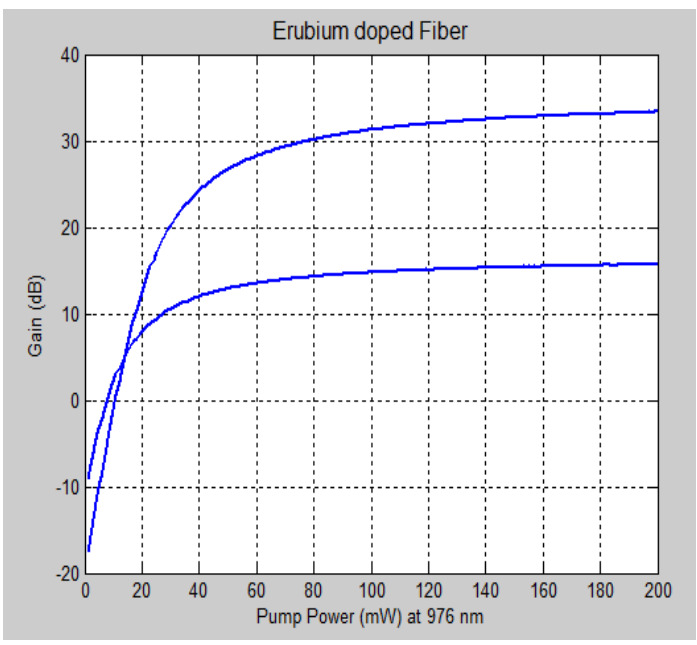

Figure 7: Graph between the Gain and Pump power

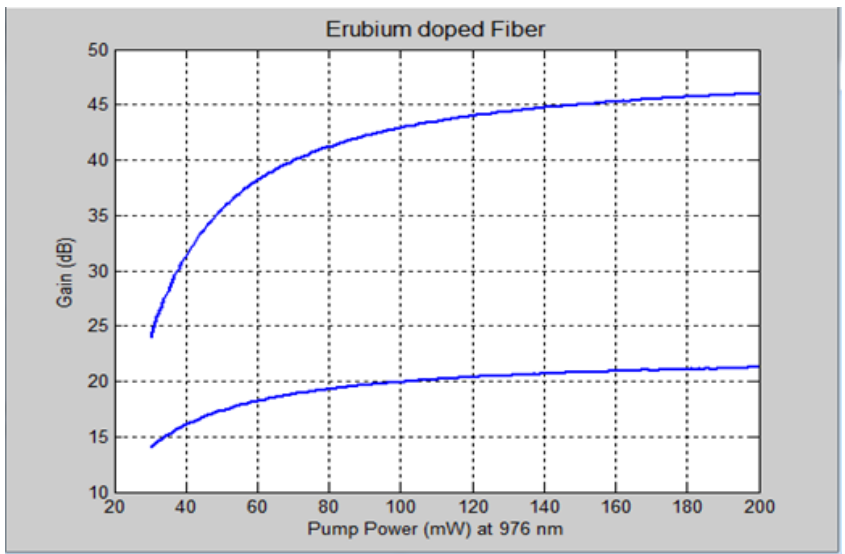

Figure 8: Gain is plotted against wavelength at different fiber length

Pump Wavelength for simulation is the 976nm.Combination of the logic is shown by the A, B, C, $\mathrm{D}, \mathrm{E}, \mathrm{F}, \mathrm{G}, \mathrm{H}, \mathrm{I}, \mathrm{K}$ and N. A= EDF0 - EDF1 this is logical combination of A. $\mathrm{B}=\mathrm{EDF} 0$ - EDF2 this is logical combination of $\mathrm{B}$. $\mathrm{C}=\mathrm{EDF} 0$ - EDF3 this is logical combination of $\mathrm{C}$. $\mathrm{D}=\mathrm{EDF} 0$ - EDF4 this is logical combination of D. E=EDF0 - EDF1 - EDF2 this is logical combination of E. F=EDF0 - EDF1 - EDF3 this is logical combination of $\mathrm{F}$. G=EDF0 - EDF1 - EDF4 this is logical combination of G. H=EDF0 - EDF2 - EDF3 this is logical combination of $\mathrm{H}$. I=EDF0 - EDF3 - EDF4 this is logical combination of I. J=EDF0 - EDF1 - EDF2 - EDF3 this is logical combination of J. K=EDF0 - EDF1 - EDF2 - EDF4 this is logical combination of $\mathrm{K} . \mathrm{M}=\mathrm{EDF} 0$ - EDF1 - EDF3 - EDF4 this is logical combination of M. N=EDF0 - EDF2 - EDF3 - EDF4 this is logical combination of N. P=EDF0 EDF1 - EDF2 - EDF3 - EDF4 this is logical combination of $P$. From above combination we can examine that as EDF length increases gain increases and the gain is increase by using the logic of the EDF combinations.

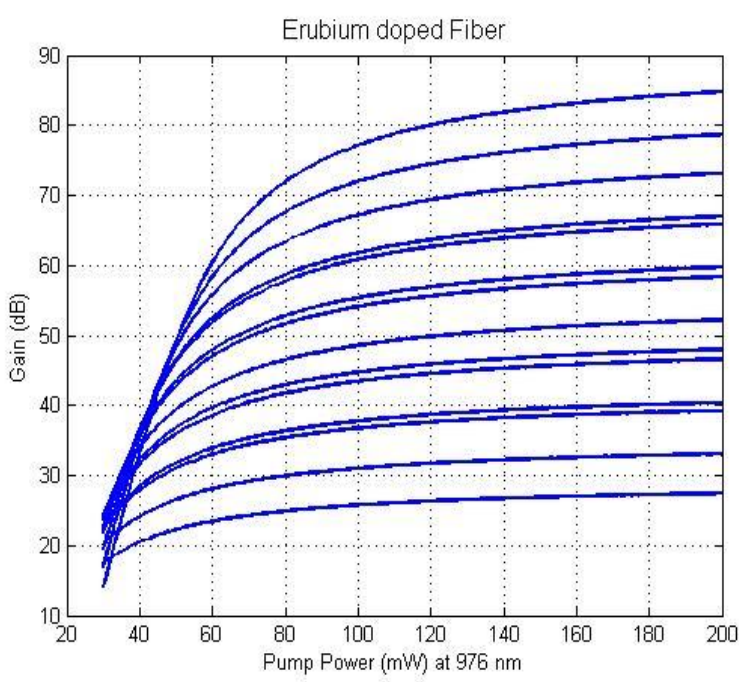

Figure 9: Graph between Gain and Pump Power by using switches

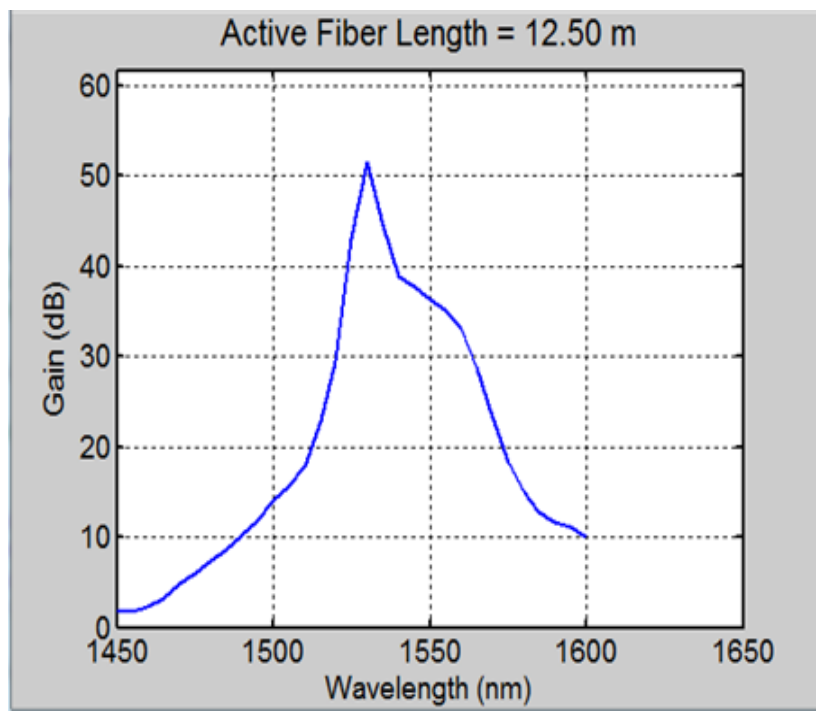

Figure 10: Graph between the Gain and wavelength

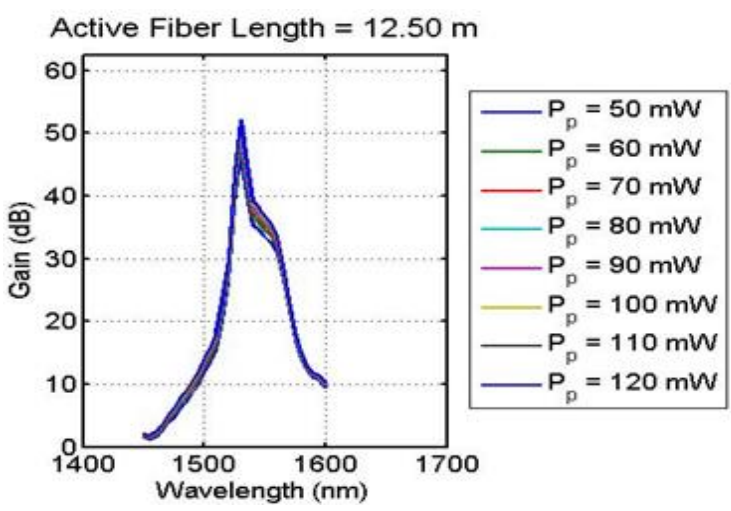

Figure 11: Graph between the Gain and wavelength using the switches

Figure 10 and 11 shows the gain spectra for various input signal powers. The spectra were measured by scanning the gain bandwidth with a low-power probe signal while inputting four WDM channels located at $1400 \mathrm{~nm}-1700 \mathrm{~nm}$. The probe power is $50 \mathrm{~mW}$ to $120 \mathrm{~mW}$, the power of each WDM channel is different. Figure 10 
shows simulation consisted of active fiber length is $12.50 \mathrm{~m}$ and gain versus wavelength of the range 1450 to $1650 \mathrm{~nm}$. There are Spontaneous decay rate of excited ions is 0.008 and Quantum efficiency of pump is $85 \%$. Figure 11 shows, that the Gain and the wavelength at the different pump powers. At the gain is increase at the 50$60 \mathrm{~dB}$ and the wavelength 1400-1700 nm.
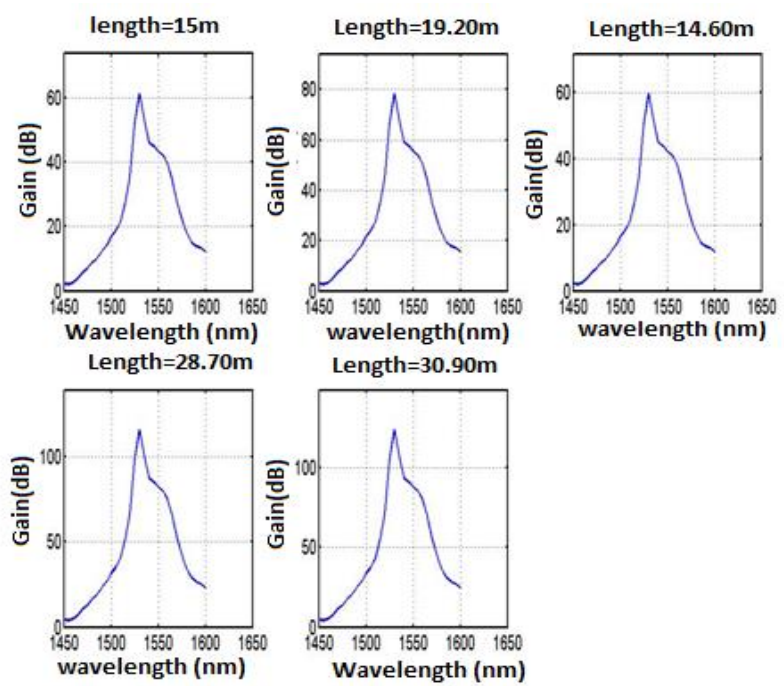

Figure 12: Graph between the Gain and Wavelength by using more switches

In Figure 12 shows Graph between the Gain and the wavelength at the different pump powers. At the gain is increase at the 50-60 dB and the wavelength 1450-1650 $\mathrm{nm}$. There are length of the fiber is $15 \mathrm{~m}, 19.20 \mathrm{~m}, 14.60$ $\mathrm{m}, 28.70 \mathrm{~m}$ and $30.90 \mathrm{~m}$. When increase the length then the gain of the fiber is also increase. This is graph is by the using $5 \mathrm{EDF}$ amplifiers i.e. more number of amplifiers.

In this paper, increase the gain and reduce the noise figure of the system. The EDFA is a key of WDM optical transmission systems. Since each period in a transmission system has a different attenuation, the EDFA gain must change according to the optical power level input into the amplifier. In such an EDFA, the population inversion level averaged along the EDF is kept constant to maintain a flat gain. Therefore, a typical variable gain amplifier consists of two and four amplifier stages with a VOA inserted between them .The total average inversion level of the two amplifier stages is kept constant and variable gain is achieved by changing the VOA attenuation. An amplifier with such a two stage amplifier configuration suffers from an increased NF in the low gain region, which is caused by the additional attenuation of the VOA. The NF degradation limits the gain range and results in the need for a large number of amplifier inventories with different gain ranges, EDFA is used for to reduce the NF and increased the Gain of the system because it is operate in the $\mathrm{C}$ band configuration.

\section{CONCLUSION}

In this Paper, proposed and demonstrated the successful information of commercial WDM system and a brief introduction to the EDFA that is the most important components in WDM communications, enabled by practical EDFAs, has it turn fuelled the developed of high power, wide bandwidth, low noise, gain flattened optical amplifiers. The important issues related to the amplifier performance, namely the optical noise figure and bandwidth to increase the Gain. Optical system can achieve much higher data rates than electronic system. The most important factors increased the transmission distance of fiber optical communication systems length and wide network area also, the optical power loss caused by scattering and absorption mechanisms in optical fiber is occur that is reduced by the mechanism of the WDM and EDFA.

The EDFA gain must change according to the optical power level input into the amplifiers. To avoid excess NF degradation, a variable gain EDFA has been proposed that modifies the EDF length by using an optical switch method. However, the variable gain EDFA in assumes the use of a single $2 \times 2$ bulk switch, $1 \times 2$ and $3 \times$ 2 bulk switches also, to modify the EDF length since the use of many optical bulk switches is impractical.

The concept and advantage of the SW-EDFA in terms of NF and pump power qualitatively and analyzed the NF and the pump power characteristics using the gain $85 \mathrm{~dB}$ and the NF is $6.5 \mathrm{~dB}$. The numerical calculation shows that an SW-EDFA with five EDFs whose combination can be changed by using SWs exhibits less NF change and requires less pump power than the conventional two and four stage EDFAs for a variable gain range of $85 \mathrm{~dB}$. We confirmed that we realized better characteristics as regards NF variation and the required pump power of the SW-EDFA than those obtained with the conventional two and four stage EDFAs. The SWEDFA exhibited a smaller NF variation of $6.5 \mathrm{~dB}$ for a variable gain of $20 \mathrm{~dB}$ and $30 \mathrm{~dB}$ for two and four stages at the time NFis $12 \mathrm{~dB}$ and $8.5 \mathrm{~dB}$.

This paper presents a comparative study on variable gain fiber of EDFA amplifier with the switching method. The EDFA is change the total fiber network length by selecting six combinations of the noise figure and pump power. The relation between pump power and amplifier gain at the different wavelength \& at the different doping concentration. The purpose is to increase the gain and reduce the noise figure of the fiber. 


\section{REFERENCES}

[1] Takaaki Mukai, Yoshihisa Yamamoto, Tatsuya Kimura,"S/N and Error Performance in AlGaAs Semiconductor Laser Preamplifier and Linear Repeater Systems", IEEE Transaction on Microwave Theory and Techni Ques,vol. 30, no. 10, 1982, pp. 1548-1556.

[2] M.C. Farries, P.R. Morkel, R.I. Laming, T.A. Birks, D.N. Payne and E.J. Tarbox, “ operation of Erbium Doped Fiber Amplifier and lasers pumped with Frequency- Doubled Nd: YAG Lasers", Journal of Lightwave Technology, vol. 7, no. 10, 1989, pp. 1473-1477.

[3] E. Desurvire and J.R. Simpson, "Amplification of Spontaneous Emission in Erbium-Doped Single-Mode Fibers", J. Lightwave Tech., Vol.7, 1989, No.5, 835.

[4] A. A. M. Saleh, Fellow, R.M. Jopson, J.D. Evankow and J.Aspell, “ Modeling of Gain in Erbium- doped Fiber Amplifiers”, IEEE Photonics Technology letters, vol. 2, 1990, no. 10, pp.714-71.

[5] R. J. Mears, S. R. Baker, "Erbium Fiber Amplifiers and Lasers", Optical and Quantum Electronics, Vol.24, 1992, PP. 517.

[6] A.K. Srivastava, Y. Sun, J.L. Zyskind, J.W. Sulhoff, , “ EDFA Transient Response to Channel Loss in WDM Transmission System", IEEE Photonics Technology letters, vol. 9, no. 3, 1997, pp. 386-388.

[7] P. C. Becker, N. A. Olsson, and J. R. Simpson, "Erbium-doped fiber amplifiers: fundamentals and technology", Academic Press, vol.3, 1997, no. 15 , pp 1-5.

[8] L. Tan Cevski, L. A. Rusch, and A. Bononi, "Gain Control in EDFA's by Pump Compensation", IEEE Photonics Technology Letters, vol. 10, no. 9, 1998, pp. 1550

[9] Yan Sun, Senior, J.L. zyskind, A.K.Srivastava, "Average Inversion level, Modeling and Physics of Erbium Doped Fiber Amplifiers", IEEE Jouranal of Lightwave Technology, vol.3, no. 4, 1999, pp. 991-1007.

[10] P.C. Becker, N.A. Olsson, and J.R. Simpson, "Erbium-Doped Fiber Amplifiers", Academic Press, 1999.

[11] W. Johnstone, B. Culshaw, D. Walsh, D. Moodie, and I. Mauchline,"Student Laboratory Experiments on Erbium Doped Fiber Amplifiers and Lasers", SPIE Proceedings, 6th International Conference on Education on Training of Optics and Photonics, 2000, Vol. 3831, 259.

[12] A. Carena, V. Curri and P. Poggiolini, " On the Optimization of Hybrid Raman/ Erbium- doped Fiber Amplifiers", IEEE Photonics Technology, vol. 13, no. 11, 2001, pp. 1170-1172.

[13] Stephanie Novak and Richard Gieske, " Simulink Model for EDFA Dynamics applied to Gain Modulation", Journal of Lightwave Technology, vol. 20, no. 6, 2002, pp. 986-992.

[14] G.P Agrwwal,"Fiber-Optic Communication Systems", Wiley Interscience, 3rd ed., 2002.

[15] M. Melo, O. Frazao, A.L.J. Teixeira, L.A. Gomes, J.R. Ferreira D. Rocha, H.M. Salgado, "Tunable L-band erbium-doped fiber ring laser by means of induced cavity loss using a fiber taper", Applied Physics B, 2003, Vol.77, 139.

[16] M.A. Othman, M.M. Ismail, M.H. Misran, M.A.M. Said and H.A Sulaiman , " Erbium Doped Fiber Amplifier (EDFA) for C- Band optical Communication System", International Journal of Engineering \& Technology IJET- IJENS, vol. 12, no. 04, 2012, pp. 48-50.

[17] Hirotaka Ono, Member, Toshio Watanabe, Tetsuo Takahashi, and Tadashi Sakamoto, Kenya Suzuki,Atsushi Mori,"Wide-Range Variable Gain Fiber Amplifier With Erbium- Doped Fiber Switching", Journal of Light wave Technology, Vol. 31, No. 12, June 15 2013, PP. 1965-1972.

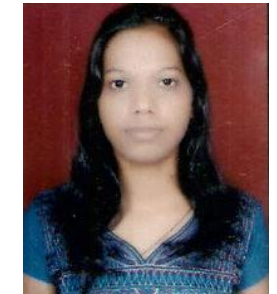

Shikha Gautam is Bron on 2nd, 1990 in Moradabad (U.P.). She is hardworking, systematic, creative with a will to learn and with a ability to grasp things fast, enjoy workinga challenging environment with confidence in her knowledge, enjoy leading teams to attain goal or to organize event set, having a desire to learn about new technologies and to work on them. She is B.Tech (Electronics \& Communication Engineering)from Moradabad Institute of Technology Moradabad, U.P. Currently she is pursuing Masters of Technology (Electronics \& Communication Engineering)from Ajay Kumar Garg Engineering College, Ghaziabad, U.P.

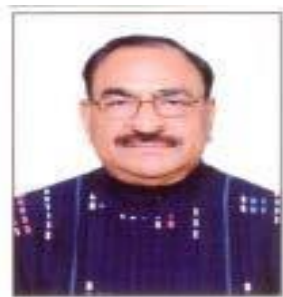

Prof. R. L. Sharma obtained M.Tech degree in Optoelectronics and Optical Fiber communication from

Indian institute of technology Delhi (India) and $\mathrm{Ph} . \mathrm{D}$. degree from Singhania University. He has about 35 years of rich experience in maintaining, supervising and managing large communication Networks involving HF, VHF, UHF and Data communication spread over the entire country, particularly far-flung and remote are as of northeastern regions and Jammu \& Kashmir of India.Forthepast5years, hehasbeenteaching optical fiber communication, Digital communication and optical networks in the department of ECE at A K G Engineering College, Ghaziabad, UP, INDIA. His research area is in the field of optical communication. 